\title{
Identification of Pold2 as a novel interaction partner of protein inhibitor of activated STAT2
}

\author{
YING ZHENG $^{1}$, SHUDONG SHENG ${ }^{2}$, HAIYAN WANG ${ }^{1}$, XIAOQING JIA ${ }^{1}$, \\ YANQIU HU $^{3}$, YAYUN QIAN ${ }^{1}$, YONGZE ZHU ${ }^{1}$ and JIANJUN WANG ${ }^{1}$ \\ ${ }^{1}$ Department of Histology and Embryology, Medical College, Yangzhou University; ${ }^{2}$ Department of Medicine, \\ Yangzhou Vocational College of Environment and Resources; ${ }^{3}$ Center of Reproductive Medicine, \\ Clinical Medical College, Yangzhou University, Yangzhou 225001, P.R. China
}

Received February 18, 2012; Accepted June 20, 2012

DOI: $10.3892 /$ ijmm.2012.1065

\begin{abstract}
Pold 2 is a subunit of the DNA polymerase $\delta$ complex, encoding a protein involved in DNA replication and repair. In this study, using a yeast two-hybrid screening technique and the common cDNA fragment of the mouse PIAS2 as a bait, Pold2 was found to interact with PIAS2. A direct interaction between Pold 2 and PIAS2 was confirmed by direct yeast two-hybrid. In vivo evidence of Pold 2 association with PIAS2 was obtained by co-immunoprecipitation using HEK-293 cells. Subcellular localization studies demonstrated that Pold2 and PIAS2 were partially co-localized in mammalian cells. Collectively, our results suggest that Pold 2 interacts under physiological conditions with PIAS2.
\end{abstract}

\section{Introduction}

DNA replication is essential not only for duplication of the genome but also for maintenance of genomic integrity during DNA repair $(1,2)$. Chromosomal DNA replication in eukaryotic cells requires three distinct DNA polymerases- $\alpha,-\delta$ (Pol $\delta$ ), and $-\varepsilon(1-5)$. Mammalian Pol $\delta$ plays a crucial and versatile role in DNA replication and various DNA repair processes (6). Its function as a chromosomal DNA polymerase is dependent on the association with proliferating cell nuclear antigen (PCNA) which functions as a molecular sliding clamp $(7,8)$. Similar to $S$. pombe enzyme, the mammalian Pold consists of four subunits: p125, p50 (also named DNA polymerases $\delta 2$ small subunit, Pold2), p66 and p12, forming a heterotetrameric complex $(4,9)$. It has been reported that Pold2 serves as a scaffold for the assembly of Pol $\delta$ by interacting simultaneously with all of the other three subunits (10). In addition, Pold2 is also

Correspondence to: Dr Ying Zheng, Department of Histology and Embryology, Medical College, Yangzhou University, Yangzhou 225001, P.R. China

E-mail: yzzkl@163.com

Key words: yeast two-hybrid system, protein inhibitor of activated STAT2, Pold2, protein interaction involved in the recruitment of several proteins regulating DNA metabolism, including p21, PDIP1 (polymerase $\delta$-interacting protein 1, PDIP1), PDIP38, PDIP46 and WRN (Werner protein, WRN) (11-14).

PIAS2 [protein inhibitor of activated STAT (signal transducer and activator of transcription) 2, PIAS2] belongs to the family of PIAS proteins, which includes PIAS1, PIAS2, PIAS3 and PIASy (15-18). PIAS proteins were initially identified as specific cofactors inhibiting DNA binding and transcriptional activation by the STAT family of transcription factors (15). These proteins typically contain an N-terminal SAP domain, an N-terminal PINIT domain, a central RING-like zincfinger (RLD) domain and a C-terminal acidic domain (19,20). The human PIAS2 gene encodes two splice variants, PIASx $\alpha$ (also called ARIP3, androgen receptor-interacting protein 3) and PIASx $\beta$ (also known as Miz1, Msx-interacting-zinc finger) (16,21). The mouse PIAS2 gene has at least five isoforms (isoforms 1-5), which differ in the $\mathrm{N}$ - and $\mathrm{C}$-termini as a result of alternative splicing. The mouse PIAS2 protein shows $99.4 \%$ sequence identity to the human homologue. Although PIAS2 was originally identified as a protein that binds to and inhibits STAT4, the functions of PIAS2 protein are clearly not limited to the regulation of STAT4. PIAS2 is capable of interacting with and modulating (activating and repressing) transcriptional activities of several transcription factors (22-24).

To determine the molecular mechanism of PIAS2 function, we used the yeast two-hybrid screening technique to identify interacting proteins associated with PIAS2. Interestingly, Pold2 was found to interact with PIAS2. Here we report evidence for a direct interaction between Pold2 and PIAS2.

\section{Materials and methods}

Yeast two-hybrid analysis. The common cDNA fragment of the mouse PIAS2 gene [9-401 aa corresponding to nucleotides (nt) 213-1391 of NM_008602] was cloned into the pGBKT7 vector containing the GAL4 DNA-binding domain to generate the bait plasmid, pGBKT7-PIAS2. This construct did not show toxic effects or autonomous transcriptional activation following transformation into the yeast strain, Y187. The mouse stem cell cDNA library was constructed in a pGADT7-Rec vector 
containing a GAL4 activation domain using Matchmaker Library Construction and Screening kits (Clontech, Santa Clara, USA) and then transformed into the yeast AH109 strain. Yeast two-hybrid screening was performed using the Matchmaker Two-Hybrid system (Clontech). Positive clones were selected based on their ability to grow on synthetic dropout (SD) medium/-LTHA/X- $\alpha-$ Gal and for X- $\alpha-G a l$ activity.

Clone isolation and interaction analysis. Plasmid DNA of prey clones was isolated (Qiagen, Hilden, Germany) and transformed into $E$. coli $\mathrm{DH} 5 \alpha$. Prey clones were recovered by ampicillin resistance and cDNA inserts were identified by PCR amplification, sequencing, and BLAST alignment. Interaction between the bait and identified prey clones was verified by co-transforming the purified prey plasmid with the bait pGBKT7-PIAS2 construct into the yeast AH109 strain followed by selection on SD/-LTHA medium. Co-transforming of pGBKT7-p53 with pGADT7-SV40 was used as a positive control. A negative control was also included by co-transforming of pGBKT7-p53 with pGADT7 vector into the same yeast cells.

Expression constructs and antibody preparation. The common cDNA fragment of the mouse PIAS 2 gene was amplified by PCR and cloned into the pCMV-Myc and pDsRed-Express-1 vector (Clontech) to generate pCMV-c-Myc-PIAS2 and pDsRedExpress-1-PIAS2 respectively. Full-length Pold2 was cloned into pEGFP-N1 (Clontech) to generate pEGFP-N1-Pold2.

The following commercially available antibodies were used for immunoprecipitation and western blot analysis: monoclonal anti-c-Myc antibody (Millipore Biotechnology, Bedford, MA, USA) and rabbit anti-GFP polyclonal antibody (Epitomics, Burlingame, USA).

Cell culture and transfection. HEK 293T and HeLa cells (human epithelial carcinoma line) were cultured and maintained in DMEM (Invitrogen) supplemented with $10 \%$ fetal bovine serum at $37^{\circ} \mathrm{C}$ in a humidified atmosphere containing $5 \% \mathrm{CO}_{2}$. Transient transfection of HEK 293T and HeLa cells (75-90\% confluence) was performed using Lipofectamine 2000 (Invitrogen, Carlsbad, CA, USA) according to the instructions provided by the manufacturer.

Immunoprecipitations. HEK 293T cells were transiently transfected with pCMV-c-Myc-PIAS2 and pEGFP-N1-Pold2 constructs and maintained as described above for $24 \mathrm{~h}$. The cells were then washed with ice-cold PBS and harvested in $500 \mathrm{ml}$ lysis buffer $[150 \mathrm{mM} \mathrm{NaCl}, 50 \mathrm{mM}$ Tris $\mathrm{pH} 8.0,1 \%$ Nonidet P-40, 0.5\% deoxycholate, and a protease inhibitor mixture (Roche Applied Science, Mannheim, Germany)]. After incubation in lysis buffer for $60 \mathrm{~min}$ on ice, lysates were clarified by centrifugation for $20 \mathrm{~min}$ at $14,000 \mathrm{rpm}$ at $4^{\circ} \mathrm{C}$. Specific monoclonal antibodies $(8 \mu \mathrm{g})$ were added to the supernatant and incubated for $120 \mathrm{~min}$ at $4^{\circ} \mathrm{C}$ prior to addition of $50 \mu \mathrm{l}$ protein $\mathrm{G}$-agarose followed by an overnight incubation at $4^{\circ} \mathrm{C}$. Samples were then centrifuged for $1 \mathrm{~min}$ in a microcentrifuge and washed with $1 \mathrm{ml}$ lysis buffer, $1 \mathrm{ml}$ washing buffer $2(500 \mathrm{mM} \mathrm{NaCl}$, $50 \mathrm{mM}$ Tris $\mathrm{pH} 7.5,0.1 \%$ Nonidet P-40, $0.05 \%$ deoxycholate) and $1 \mathrm{ml}$ washing buffer $(10 \mathrm{mM}$ Tris $\mathrm{pH} 8.0,0.1 \%$ Nonidet P-40, $0.05 \%$ deoxycholate).
Western blot analysis. Initial lysates and immunoprecipitated proteins were analyzed by SDS-PAGE and immunoblotting was carried out using specific antibodies. Proteins were electrophoretically transferred to nitrocellulose membranes (Amersham Biosciences, Amersham, UK) and probed with the appropriate antibodies. Bound antibodies were visualized with horseradish peroxidase (HRP)-conjugated anti-rabbit or anti-mouse (Sigma) IgG antibodies using the enhanced chemiluminescence system (ECL, Thermo Scientific, MA, USA).

Fluoresence microscope analysis. To detect PIAS2 and Pold2, HEK 293T/HeLa cells plated on round coverslips were transiently transfected with the pDsRed-Express-1-PIAS2 and pEGFP-N1-Pold 2 constructs using Lipofectamine 2000 reagent. After 48 h, cells were washed twice with PBS and visualized by Nikon scanning laser confocal microscope.

\section{Results}

Identification of Pold2 as a PIAS2-interacting protein using a yeast two-hybrid screening system. To identify mouse proteins that interact with PIAS2, cDNA libraries derived from mouse stem cells were screened using a yeast two-hybrid system with the bait plasmid pGBKT7-PIAS2. Growth of yeast strain Y187 co-transformed with this construct and the empty prey plasmid pGADT7 was not supported on SD/-LTHA medium confirming that the bait was not auto-activated. A total of $2 \times 10^{6}$ primary transformants were screened, yielding over 54 positive clones, 10 of which demonstrated strong growth on SD/-LTHA medium. These clones were isolated, sequenced and aligned using the NCBI BLAST alignment search tool. One clone encoded Pold2 (DNA polymerase, $\delta 2$ small subunit), spanning regions of 177 amino acids (Pold2 292-469 aa) was found to interact with PIAS2 protein. To confirm these results, the PIAS2-Pold2 interaction was further investigated using a direct yeast hybrid screening system. The yeast strain co-transformed with pGADT7-Pold2 and pGBKT7 could not grow on SD/-LTHA medium and showed negative $\beta$-galactosidase activity (Fig. 1B, clone 3 ). While strong growth on SD/-LTHA medium was observed only in yeast cells co-transformed with pGBKT7-PIAS2 and pGADT7-Pold2 (Fig. 1B, clone 1), indicating interaction of Pold 2 with PIAS2 in yeast.

Pold2 interacts with PIAS2 in vivo. The data obtained from the yeast two-hybrid screen was confirmed by evidence of a direct interaction between PIAS 2 and Pold 2 in mammalian cells. The expression vectors for c-myc-tagged PIAS2 and GFP-tagged Pold2 were co-transfected to human embryonic kidney 293T cells. Twenty-four hours after transfection, the cell extract was prepared, and the proteins in the extract were first immunoprecipitated with the anti-c-myc, anti-GFP and non-specific IgG, respectively. The precipitates were then immnuoblotted against the anti-GFP or anti-c-myc antibodies, respectively (Fig. 2). The anti-c-myc antibody precipitated c-myc-PIAS2. GFP-Pold2, on the other hand, was detected in the immunoprecipitate with the ant-GFP antibody but not with $\mathrm{IgG}$ (Fig. 2). PIAS2 protein was also immunoprecipitated from the transfected HEK 293T cells using a rabbit anti-GFP antibody prior to detection of PIAS2 by anti-c-myc antibody. As shown in Fig. 2, Pold2 and PIAS2 fusion proteins were observed in 
A

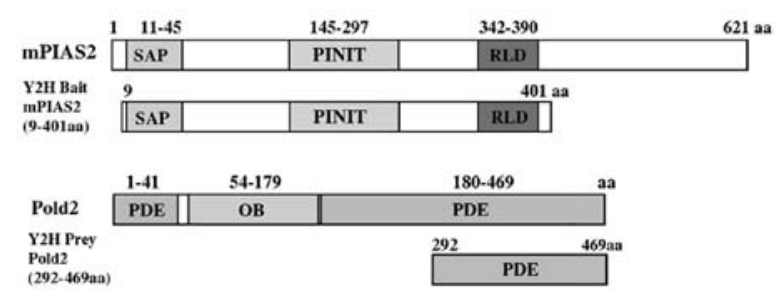

B

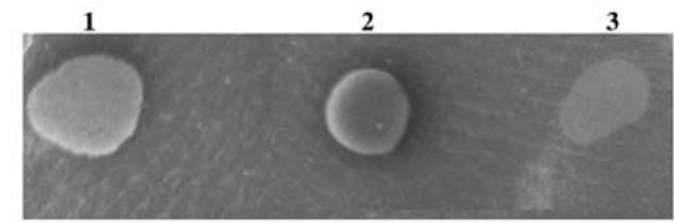

Figure 1. Yeast two-hybrid bait and prey constructs and direct yeast two-hybrid analysis. (A) Yeast two-hybrid bait construct. The common cDNA fragment of the mouse PIAS2 gene [9-401 aa corresponding to nucleotides (nt) 213-1391 of NM_008602] was cloned into the pGBKT7 vector containing the GAL4 DNA-binding domain to generate the bait plasmid, pGBKT7-PIAS2. The prey of Pold2 protein (from 292 to 469 aa) was identified. (B) Direct yeast twohybrid analysis was used to confirm the interaction between Pold2 and PIAS2. Clone 1 was Pold2 prey co-transformed with the PIAS2 plasmid, clone 2 was the positive control (pGBKT7-p53 and pGADT7-SV40 antigen), and clone 3 was the negative control (pGADT7-Pold2 and pGBKT7).
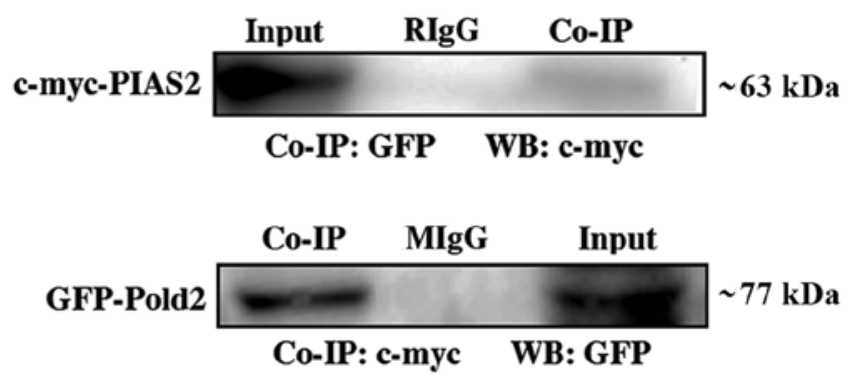

Figure 2. PIAS2 co-immunoprecipitates with Pold2. HEK 293 T cells were homogenized in lysis buffer. Then the cell lysates were incubated with anti-GFP antibody, anti-c-myc antibody and non-specific IgG for immunoprecipitation (IP), respectively. Co-immunoprecipitants (Co-IP) were analyzed by western blotting (WB) with anti-c-myc and anti-GFP antibodies, respectively. The total lysates (input) were also analyzed.

the co-immunoprecipiate and total lysate (input). These results indicated that Pold2 can interact with PIAS2 in mammalian cells.

Co-localization of Pold2 with PIAS2 in HEK $293 \mathrm{~T}$ and HeLa cells. To further confirm the interaction between Pold2 and PIAS2 proteins, the co-localization was demonstrated by co-transfected pDsRed-Express-1-PIAS2 and pEGFP-N1-Pold2 into HEK 293T and HeLa cells, respectively. Two days after transfection, the cells were detected by a Nikon scanning laser confocal microscope. As shown in Fig. 3, GFP-Pold2 protein was uniformly distributed in HEK 293T and HeLa cells (Fig. 3, green) and DsRed-PIAS2 protein was expressed in these cells (Fig. 3, red). The merged image (Fig. 3, yellow) revealed that Pold2 and PIAS2 were partially co-localized in these cells, indicating that Pold2 can interact with PIAS2 in HEK 293T and HeLa cells.

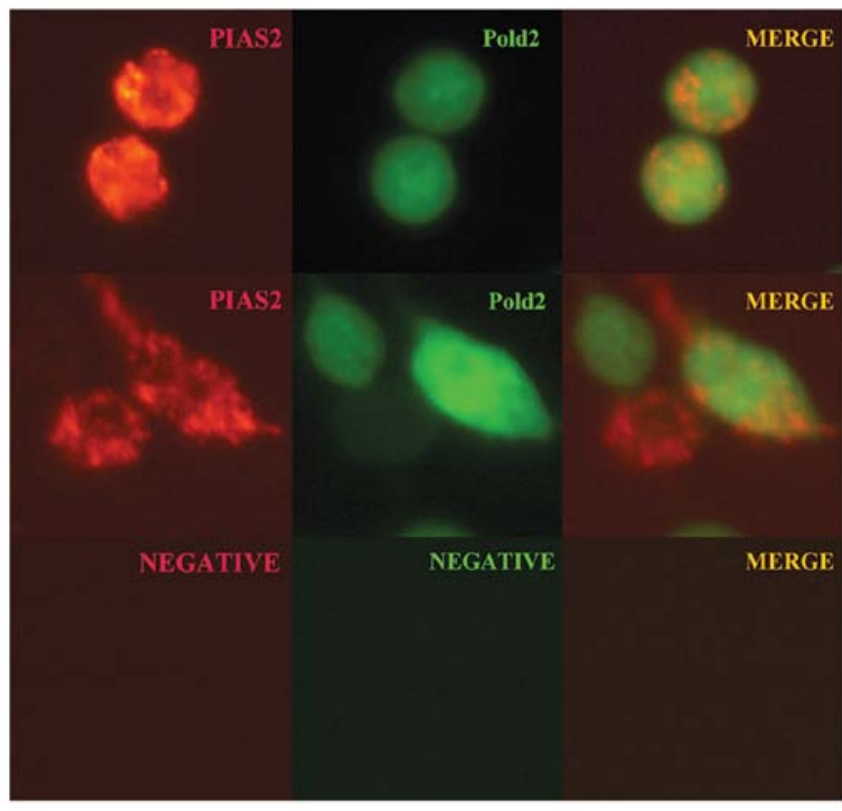

Figure 3. Co-localization of Pold2 with PIAS2 in HEK 293T and HeLa cells. HEK 293T (line 1) and HeLa cells (line 2) were transfected with expression vectors for DsRed-PIAS2 and EGFP-Pold2 by Lipofectamine 2000. Twentyfour hours after transfection, the cells were detected by fluorescence microscopy. The two figures [PIAS2 (red) and Pold2 (green)] have been merged.

\section{Discussion}

The purpose for this study was to identify novel proteins that interact with mouse PIAS2 using the yeast two-hybrid system. Human PIAS2 include PIASx $\alpha$ and PIASx $\beta$ while mouse PIAS2 gene has at least five isoforms (isoforms 1-5), which differ in the $\mathrm{N}$ - and $\mathrm{C}$-termini as a result of alternative splicing. So we used the common encoding region of mouse PIAS2 as a bait to screen a mouse cDNA library. As a result, Pold2 protein, a subunit of the DNA polymerase $\delta$ complex (10), was found to be a novel interaction partner for PIAS2.

Obviously, protein-protein interactions revealed by the yeast two-hybrid system do not necessarily indicate that the native proteins are capable of interacting, nor do they provide any evidence that such interactions take place in a cellular context. In this study, we have further characterized the interaction of Pold2 with PIAS2.

The ability of Pold2 to interact with PIAS2 was demonstrated by co-immunoprecipitation. For this purpose, both tagged forms of Pold2 and PIAS2 eukaryotic expression vectors were co-transfected into human embryonic kidney 293T cells. The whole cell extracts were used for co-immunoprecipitation. The experiments revealed that Pold2 and PIAS2 could be reciprocally co-immunoprecipitated from total cell lysates of the transfected HEK 293T cells. These studies strongly support the view that the interaction of Pold2 with PIAS2 is physiological, i.e. that this interaction takes place in a cellular context.

By using an overexpression system, we further provided evidence of a direct interaction between GFP-Pold2 and DsRed-PIAS2. Microscopy showed that Pold2 protein was distributed in the cytoplasm and nuclei of HEK 293T and HeLa cells, and PIAS2 protein expression was scattered in these cells. The merged image revealed that Pold2 and PIAS2 were partial 
co-localized in these cells. These results suggested that Pold2 can interact with PIAS2 in mammalian cells.

Mouse Pold2 was cloned from a mouse cDNA embryo library by PCR (25). It was located on chromosome 11, band A2 and encoded a 469 amino acid protein. The homology of the mouse protein with human and bovine homologues was extremely high and the interaction of the 125-kDa subunit and the 50-kDa subunit of Pold was also highly conserved between mouse and human (25). The crystal structure showed that there are three domains in Pold2: two phosphodiesterase-like (PDE) domains and one oligonucleotide/oligosaccharide binding (OB) domain. The flanking regions of Pold2 PDE provide a larger surface area and the flexibility to form many protein-protein interactions (26). In our screening, the C-terminal PDE domain of Pold2, which spanned from 292 to 469 amino acids, was found to interact with PIAS2. It has also been reported that there were many proteins interacting with Pold 2 protein and fulfilling their function. One of them is $\mathrm{p} 21^{\mathrm{Cip} / \mathrm{WAF} 1 / \mathrm{Sdi1}}(\mathrm{p} 21) \cdot \mathrm{p} 21$ was the first cyclin-dependent kinase inhibitor (CKIs) to be identified $(27,28)$. The N-terminal of $\mathrm{p} 21$ can bind to and inhibit the cyclin-dependent protein kinases (CDKs) which are necessary for $\mathrm{G} 1$ to $\mathrm{S}$ phase transition as well as $\mathrm{S}$ phase progression (29). A second cyclin binding site has also been identified near the $\mathrm{C}$ terminus of $\mathrm{p} 21$. The $\mathrm{C}$-terminal region of $\mathrm{p} 21$ was also shown to bind proliferating cell nuclear antigen (PCNA) and to inhibit DNA replication mediated by Polס (30). p21 was also found to interact with the small subunit of Pold, Pold2 (11). This ability indicated that $\mathrm{p} 21$ may be recruited to the replication complex via an interaction with Pold2. This interaction could provide the basis for the targeting or anchoring of p21 to the replication complex, thereby facilitating the actions of $\mathrm{p} 21$. The latter could include several possibilities, i.e., the inhibition of Pold by binding of p21 to PCNA within the replication complex, or by inhibition of phosphorylation of replication complex proteins by the CDK2/cyclin A kinase (11).

As a member of PIAS2, Miz1 was a mutant PIASx $\beta$ with deletion of amino acids 1-133. This mutant protein was initially described as a protein that interacts with the carboxy terminus of the Myc oncoprotein (31). Miz1 was able to activate transcription of genes encoding the cell cycle inhibitors p21, leading to cell cycle arrest (31). Induction of p21 expression required binding of Miz1 to the core $\mathrm{p} 21$ promoter and, as a consequence, factors that control Miz1 could affect p21 expression (32). Miz1 could also repress transcription when it formed complexes with other transcription factors. For instance, Miz1 could repress transcription of the p21 gene in a complex with the Myc oncoprotein. Myc repressed transcription of the p21 gene through binding to Mizl. As a result, high levels of Myc suppressed cell cycle arrest and favoured apoptosis in response to DNA damage (32). At its amino-terminus, Mizl contained a POZ protein-protein interaction domain, which can mediate both homo and heterodimerization among POZ domain proteins. Mizl heterodimerized with Zbtb4 repressed p21 expression and inhibits cell cycle arrest in response to p53 activation (33). In this study, we found that Pold 2 could interact with PIAS2. The yeast-two hybrid bait also contained the full length of Miz1 POZ domain. So there was a possibility that PIAS2 could heterodimerize with Pold2 and regulated the function of $\mathrm{p} 21$. The latter could be recruited to the Pold complex and thereby modulate signaling that could affect Pold activity. Further study is required to provide more information and evidence for understanding the functional significance of the Pold2-PIAS2 interaction.

In conclusion, evidence was presented here to demonstrate there was a direct interaction between PIAS2 and Pold2, the small subunit of Pold both in vitro and in vivo. Although its functions were currently unknown, these findings indicated that many proteins might be involved the action of Pold.

\section{Acknowledgements}

This study was supported by grants from the National Natural Science Foundation of China (no. 31071020 and no. 81000272) and the Natural Science Foundation of Jiangsu Province of China (no. BK2009192).

\section{References}

1. Hubscher U, Giovanni M and Spadari S: Eukaryotic DNA polymerases. Annu Rev Biochem 71: 133-163, 2002.

2. Sutton MD and Walker GC: Managing DNA polymerases: coordinating DNA replication, DNA repair, and DNA recombination. Proc Natl Acad Sci USA 98: 8342-8349, 2001.

3. Bell SP and Dutta A: DNA replication in eukaryotic cells. Annu Rev Biochem 71: 333-374, 2002.

4. Lee MY, Tan CK, Downey KM and So AG: Further studies on calf thymus DNA polymerase $\delta$ purified to homogeneity by a new procedure. Biochemistry 23: 1906-1913, 1984.

5. MacNeill SA, Moreno S, Reynolds N, Nurse P and Fantes PA: The fission yeast Cdcl protein, a homologue of the small subunit of DNA polymerase 8, binds to Pol3 and Cdc27. EMBO J 15: 4613-4628, 1996.

6. Pavlov YI, Shcherbakova PV and Rogozin IB: Roles of DNA polymerases in replication, repair, and recombination in eukaryotes. Int Rev Cytol 255: 41-132, 2006.

7. Mossi R and Hubscher U: Clamping down on clamps and clamp loaders-the eukaryotic replication factor C. Eur J Biochem 254: 209-216, 1998.

8. Kelman Z: PCNA: structure, functions and interactions. Oncogene 14: 629-640, 1997.

9. Xie B, Mazloum N, Liu L, Rahmeh A, Li H and Lee MY: Reconstitution and characterization of the human DNA polymerase delta four-subunit holoenzyme. Biochemistry 41: 13133-13142, 2002.

10. Li H, Xie B, Zhou Y, Rahmeh A, Trusa S, Zhang S, Gao Y, Lee EY and Lee MY: Functional roles of p12, the fourth subunit of human DNA polymerase $\delta$. J Biol Chem 281: 14748-14755, 2006.

11. Li H, Xie B, Rahmeh A, Zhou Y and Lee MY: Direct interaction of p21 with p50, the small subunit of human DNA polymerase delta. Cell Cycle 5: 428-436, 2006.

12. He H, Tan CK, Downey KM and So AG: A tumor necrosis factor $\alpha$ - and interleukin 6-inducible protein that interacts with the small subunit of DNA polymerase $\delta$ and proliferating cell nuclear antigen. Proc Natl Acad Sci USA 98: 11979-11984, 2001.

13. Liu L, Rodriguez-Belmonte EM, Mazloum N, Xie B and Lee MY: Identification of a novel protein, PDIP38, that interacts with the p50 subunit of DNA polymerase $\delta$ and proliferating cell nuclear antigen. J Biol Chem 278: 10041-10047, 2003.

14. Szekely AM, Chen YH, Zhang C, Oshima J and Weissman SM: Werner protein recruits DNA polymerase $\delta$ to the nucleolus. Proc Natl Acad Sci USA 97: 11365-11370, 2000.

15. Liu B, Liao J, Rao X, Kushner SA, Chung CD, Chang DD and Shuai K: Inhibition of Stat1-mediated gene activation by PIAS1. Proc Natl Acad Sci USA 95: 10626-10631, 1998.

16. Kotaja N, Aittomäki S, Silvennoinen O, Palvimo JJ and Jänne OA: ARIP3 (androgen receptor-interacting protein 3) and other PIAS (protein inhibitor of activated STAT) proteins differ in their ability to modulate steroid receptor-dependent transcriptional activation. Mol Endocrinol 14: 1986-2000, 2000.

17. Chung CD, Liao J, Liu B, Rao X, Jay P, Berta P and Shuai K: Specific inhibition of Stat 3 signal transduction by PIAS3. Science 278: 1803-1805, 1997.

18. Zhang C, Yuan X, Yue L, Fu J, Luo L and Yin Z: PIASy interacts with p73alpha and regulates cell cycle in HEK293 cells. Cell Immunol 263: 235-240, 2010. 
19. Sharrocks AD: PIAS proteins and transcriptional regulationmore than just SUMO E3 ligases? Genes Dev 20: 754-758, 2006.

20. Shuai K: Regulation of cytokine signaling pathways by PIAS proteins. Cell Res 16: 196-202, 2006.

21. Arora T, Liu B, He H, Kim J, Murphy TL, Murphy KM, Modlin RL and Shuai K: PIASx is a transcriptional co-repressor of signal transducer and activator of transcription. J Biol Chem 278: 21327-21330, 2003.

22. Peng Y, Lee J, Zhu C and Sun Z: A novel role for protein inhibitor of activated STAT (PIAS) proteins in modulating the activity of Zimp7, a novel PIAS-like protein, in androgen receptor-mediated transcription. J Biol Chem 285: 11465-11475, 2010.

23. Gross M, Liu B, Tan J, French FS, Carey M and Shuai K: Distinct effects of PIAS proteins on androgen-mediated gene activation in prostate cancer cells. Oncogene 20: 3880-3887, 2001.

24. Takahashi K, Taira T, Niki T, Seino C, Iguchi-Ariga SM and Arig H: DJ-1 positively regulates the androgen receptor by impairing the binding of PIASx $\alpha$ to the receptor. J Biol Chem 276: 37556-37563, 2001.

25. Hindges R and Hübscher U: Cloning, chromosomal localization, and interspecies interaction of mouse DNA polymerase delta small subunit (PolD2). Genomics 44: 45-51, 1997.

26. Baranovskiy AG, Babayeva ND, Liston VG, Rogozin IB, Koonin EV, Pavlov YI, Vassylyev DG and Tahirov TH: X-ray structure of the complex of regulatory subunits of human DNA polymerase delta. Cell Cycle 7: 3026-3036, 2008.
27. Harper J, Adami G, Wei N, Keyomarsi K and Elledge S: The p21 Cdk-interacting protein Cip1 is a potent inhibitor of G1 cyclindependent kinases. Cell 75: 805-816, 1993.

28. Noda A, Ning Y, Venable S, Pereira-Smith O and Smith J: Cloning of senescent cell-derived inhibitors of DNA synthesis using an expression screen. Exp Cell Res 211: 90-98, 1994.

29. Chen J, Jackson PK, Kirschner MW and Dutta A: Separate domains of p21 involved in the inhibition of Cdk kinase and PCNA. Nature 374: 386-388, 1995.

30. Gulbis JM, Kelman Z, Hurwitz J, O'Donnell M and Kuriyan J: Structure of the C-terminal region of $\mathrm{p} 21$ (WAF1/CIP1) complexed with human PCNA. Cell 87: 297-306, 1996.

31. Adhikary S and Eilers M: Transcriptional regulation and transformation by Myc proteins. Nat Rev Mol Cell Biol 6: 635-645, 2005.

32. Herold S, Wanzel M, Beuger V, Frohme C, Beul D, Hillukkala T, Syvaoja J, Saluz HP, Haenel F and Eilers M: Negative regulation of the mammalian UV response by Myc through association with Miz-1. Mol Cell 10: 509-521, 2002.

33. Weber A, Marquardt J, Elzi D, Forster N, Starke S, Glaum A, Yamada D, Defossez PA, Delrow J, Eisenman RN, Christiansen H and Eilers M: Zbtb4 represses transcription of P21CIP1 and controls the cellular response to p53 activation. EMBO J 27: 1563-1574, 2008. 